\title{
DISCOVERY OF A NEW MAYFLY SPECIES (EPHEMEROPTERA, BAETIDAE) NEAR CENDERAWASIH UNIVERSITY CAMPUS IN PAPUA, INDONESIA
}

\author{
Thomas Kaltenbach ${ }^{* 1,2}$, Suriani Surbakti ${ }^{3}$, Nikita J. Kluge ${ }^{4}$, Jean-Luc Gattolliat ${ }^{1,2}$, \\ Michel Sartori ${ }^{1,2}$ and Michael Balke ${ }^{5}$ \\ ${ }^{1}$ Museum of Zoology, Palais de Rumine, Place Riponne 6, CH-1005 Lausanne, Switzerland \\ ${ }^{2}$ University of Lausanne (UNIL), Department of Ecology and Evolution, CH-1015 Lausanne, Switzerland \\ ${ }^{3}$ Department of Biology, Cenderawasih University (UNCEN), Jl. Kumbolker Perumnas III, Yabansai, Heram, \\ Kota Jayapura, Papua 99224, Indonesia \\ ${ }^{4}$ Department of Entomology, Biological Faculty, Saint-Petersburg State University, \\ Universitetskaya nab., 7/9, Saint Petersburg, 199034, Russia \\ ${ }^{5}$ SNSB-Zoologische Staatssammlung München (ZSM), Münchhausenstrasse 21, D-81247 München, Germany \\ *Corresponding author: thomas.kaltenbach@bluewin.ch
}

\begin{abstract}
Material collected just behind the Cenderawasih University campus in Jayapura, Papua Province, Indonesia, revealed a new species of the Labiobaetis claudiae group, which is here described and illustrated based on larvae, subimago, male and female imagos. The total number of Labiobaetis species on the island New Guinea increased to 33, the total number for Indonesia increased to 26, and the total number of Labiobaetis species worldwide is augmented to 147. A key to the larvae of the $L$. claudiae group is provided. The interspecific K2P distances between species of the L. claudiae group are between $20 \%$ and $23 \%$.
\end{abstract}

Keywords: Integrative taxonomy, New Guinea, species discovery

\begin{abstract}
ABSTRAK
Spesies baru dari kelompok Labiobaetis claudiae diungkap berdasarkan spesimen yang dikumpulkan dari belakang kampus Universitas Cenderawasih di Jayapura, Provinsi Papua, Indonesia. Spesies baru dideskripsikan dan diilustrasikan berdasarkan larva, subimago, imago jantan, dan betina. Jumlah total spesies Labiobaetis di pulau Papua meningkat menjadi 33, jumlah total untuk Indonesia meningkat menjadi 26, dan jumlah total spesies Labiobaetis di seluruh dunia bertambah menjadi 147. Kunci identifikasi larva untuk kelompok $L$. claudiae disediakan. Jarak antar spesies K2P antar spesies dari kelompok L. claudiae adalah antara $20 \%$ dan $23 \%$.
\end{abstract}

Kata kunci: Taksonomi integrative, Papua, penemuan spesies baru

\section{INTRODUCTION}

The family Baetidae has the highest species diversity among mayflies, comprising 1,070 species in 110 genera (Sartori \& Brittain, 2015; Jacobus et al., 2019), which is approximately one quarter of all mayfly species worldwide (Gattolliat \& Nieto, 2009; Cruz et al., 2020). They have a cosmopolitan distribution except Antarctica, New Zealand, New Caledonia and a few remote islands. The genus Labiobaetis Novikova \& Kluge (Novikova \& Kluge, 1987) is one of the richest genera of mayflies with 146 named species (Kaltenbach et al., 2020 and citations therein, Kaltenbach \& Gattolliat, 2021a, b). Recently, 65 new species were described from the highly diverse and poorly explored Southeast Asia and New Guinea regions, based 
on integrative taxonomy (Kaltenbach \& Gattolliat, 2018, 2019, 2020; Kaltenbach et al., 2020). Labiobaetis has a nearly worldwide distribution, excluding only the Neotropical realm, New Zealand and New Caledonia. The history and concept of the genus Labiobaetis were recently summarized in detail (Shi \& Tong, 2014; Kaltenbach \& Gattoliat, 2018).

The diversity of Labiobaetis in New Guinea was previously studied in two papers (Lugo-Ortiz et al., 1999; Kaltenbach \& Gattolliat, 2018) and a total of 32 species were described. Here, we describe another New Guinea species of the Labiobaetis claudiae group based on larvae, subimago, male and female imagos. So far, this group was known from larvae of two species, L. claudiae Kaltenbach \& Gattolliat, 2018 and L. stagnum Kaltenbach $\&$ Gattolliat, 2018. The group is well distinguished from other groups or species not assigned to a group by the following characters: labial palp segment II with a narrow thumb-like distomedial protuberance, segment III rather large; maxillary palp apically constricted, without excavation at segment II; six pairs of tergalii, margin of tergalii with both longer and shorter setae; hind protoptera absent; scape process absent; femoral patch present on all legs; setae on dorsal margin of femur short (0.12-0.16x maximum width of femur).

\section{MATERIALS AND METHODS}

The larvae were collected by kick-sampling by a team of Cenderawasih University (UNCEN) staff and visiting scientists during field practicals, and preserved in $96 \%$ ethanol.

Subimagines were reared from mature larvae in cages placed in the river. Subsequently, imagines of both sexes were reared from subimagines placed in containers with wet air, but without water. Imagos and subimagos were individually associated with larval and subimaginal exuviae.

The dissection of larvae was done in Cellosolve (2-Ethoxyethanol) with subsequent mounting on slides with liquid Euparal, using an Olympus SZX7 stereomicroscope.

The DNA of part of the specimens was extracted using non-destructive methods allowing subsequent morphological analysis (see Vuataz et al., 2011 for details). We amplified a 658 bp fragment of the mitochondrial gene cytochrome oxidase subunit 1 (COI) using the primers LCO 1490 and HCO 2198 (Folmer et al., 1994, see Kaltenbach \& Gattolliat 2020 for details). Sequencing was done with Sanger's method (Sanger et al., 1977). The genetic variability between specimens was estimated using Kimura-2-parameter distances (K2P) (Kimura, 1980), calculated with the program MEGA 7 (Kumar et al., 2016; http:// www.megasoftware.net). 
Drawings were made using an Olympus BX43 microscope. In order to facilitate the determination and the comparison of important structures, we partly used a combination of dorsal and ventral aspects in one drawing. Explanations are given in Kaltenbach et al., 2020: fig. 1 .

Photographs were taken using a Canon EOS 6D camera and other digital cameras and processed with the programs Adobe Photoshop Lightroom (http://www.adobe.com) and Helicon Focus version 5.3 (http://www.heliconsoft.com). Photographs were subsequently enhanced with Adobe Photoshop Elements 12 and 13.

The distribution map was generated with SimpleMappr (https://simplemappr.net; Shorthouse, 2010). Google Earth (http://www.google.com/earth/download/ge/) was used to attribute approximate GPS coordinates to the sample location.

The dichotomous key was elaborated with the support of the program DKey version 1.3.0 (http://drawwing.org/dkey; Tofilski, 2018).

The terminology follows Hubbard (1995) and Kluge (2004). Consequently, the term tergalius/tergalii is used for gill/gills. For the explanations of the character states of selected larval characters, we refer to Kaltenbach et al., 2020: fig. 2.

\section{RESULTS}

Abbreviations:

KSP: Koleksi Serangga Papua, Department of Biology, Cenderawasih University (UNCEN) (Indonesia)

MZB: Museum Zoologicum Bogoriense (Indonesia)

Diagnosis of Labiobaetis claudiae group of species (larvae) (Kaltenbach and Gattoliat 2018)

Following combination of characters: A) dorsal surface of labrum with submarginal arc of simple setae; B) labial palp segment II with rather narrow thumb-like distomedial protuberance; C) maxillary palp segment II without distolateral excavation, apex constricted; D) six pairs of tergalii; E) tergalii margin with both shorter and longer setae; F) hind protoptera absent; G) distolateral process at scape absent; H) femur dorsally with relatively short setae (length $0.12-0.16 \mathrm{x}$ maximum width of femur); I) femoral patch present. 
The L. claudiae group is known from New Guinea only, with the following species:

Labiobaetis claudiae Kaltenbach \& Gattolliat, 2018

Labiobaetis stagnum Kaltenbach \& Gattolliat, 2018

\section{Labiobaetis academicus Kaltenbach, Surbakti \& Kluge sp. nov.}

Figures $1-3,5-8$

Differential diagnosis. Larva. Following combination of characters differentiate the new species from L. claudiae and L. stagnum: A) maxilla medially with 7-9 medium to long, spine-like setae; B) shape of labial palp segment II as Fig. 1h; segment III slightly pentagonal; C) shape of tergalius IV as Fig. 2d; F) paraproct distally not expanded, with 20-25 stout, marginal spines.

Description. Larva (Figs 1-3). Cerci ca. 2/3 of body length, paracercus ca. 2/3 of cerci length, antenna approx. twice as long as head length.

Coloration. Head, thorax and abdomen dorsally brown, with pattern as in Fig. 3a. Fore protoptera brown with bright striation. Head, thorax and abdomen ventrally light brown. Legs light brown; femur with a large, distomedial brown spot, dorsal margin and apex brown; tibia in basal part with brown spot, bordered by patellotibial suture. Caudalii light brown.

Antenna (Fig. 2h) with scape and pedicel subcylindrical, without distolateral process at scape.

Labrum (Fig. 1a). Rectangular, length 0.7x maximum width. Distal margin with medial emargination and a small process. Dorsally with medium, fine, simple setae scattered over surface; submarginal arc of setae composed of one plus 5-7 long, simple setae. Ventrally with marginal row of setae composed of lateral and anterolateral long, feathered setae and medial long, bifid, pectinate setae; ventral surface with ca. seven short, spine-like setae near lateral and anterolateral margin.

Right mandible (Fig. 1b, c). Incisor and kinetodontium fused. Incisor with four denticles; kinetodontium with four denticles, inner margin of innermost denticle with a row of thin setae. Prostheca robust, apically denticulate. Margin between prostheca and mola slightly convex. Tuft of setae at apex of mola present.

Left mandible (Fig. 1d, e). Incisor and kinetodontium fused. Incisor with four denticles; kinetodontium with four denticles. Prostheca robust, apically with small denticles and combshaped structure. Margin between prostheca and mola slightly convex, with minute denticles 
toward subtriangular process. Subtriangular process long and slender, above level of area between prostheca and mola. Denticles of mola apically constricted. Tuft of setae at apex of mola present.

Both mandibles with lateral margins almost straight. Basal half with fine, simple setae scattered over dorsal surface.

Hypopharynx and superlinguae (Fig. 1f). Lingua approx. as long as superlinguae. Lingua longer than broad; medial tuft of stout setae well developed, short; distal half laterally expanded. Superlinguae distally rounded; lateral margin rounded; fine, long, simple setae along distal margin.

Maxilla (Fig. 1g). Galea-lacinia ventrally with two simple, apical setae under canines. Inner dorsal row of setae with three denti-setae, distal denti-seta tooth-like, middle and proximal denti-setae slender, bifid and pectinate. Medially with one bipectinate, spine-like seta and 7-9 medium to long, simple setae. Maxillary palp approx. as long as length of galea-lacinia; 2-segmented; palp segment II 1.1x length of segment I; setae on maxillary palp fine, simple, scattered over surface of segments I and II; apex of last segment constricted, without excavation at inner distolateral margin.

Labium (Fig. 1h, i). Glossa basally broad, narrowing toward apex; shorter than paraglossa; inner margin with ca. 12 short, stout, spine-like setae increasing in length distally; apex with two long and one short, robust setae; outer margin with six spine-like setae; ventral surface with few fine, simple, scattered setae. Paraglossa sub-rectangular, curved inward; apex rounded; ventrally with three rows of long, robust, distally pectinate setae in apical area and one medium, simple seta in anteromedial area; dorsally with a row of four long, spine-like setae near inner margin. Labial palp with segment I approx. as long as segments II and III combined. Segment I ventrally with short, fine, simple setae. Segment II with narrow, thumb-like distomedial protuberance; distomedial protuberance $0.5 \mathrm{x}$ width of base of segment III; ventral surface with short, fine, simple setae; dorsally with a row of 3-6 medium, spine-like, simple setae near outer margin. Segment III slightly pentagonal; length $0.9 \mathrm{x}$ width; ventrally covered with short, spine-like, simple setae and short, fine, simple setae.

Hind protoptera absent.

Legs (Fig. 2a, b). Ratio of foreleg segments 1.3:1.0:0.6:0.2. Femur. Length ca. 3x maximum width. Dorsal margin with a row of 25-32 medium, curved, spine-like setae; length of setae $0.12 \mathrm{x}$ maximum width of femur. Apex rounded, with medium to short, 
curved, spine-like setae. Many stout, lanceolate setae scattered along ventral margin; femoral patch present. Tibia. Dorsal margin with a row of short to medium, stout, spine-like setae. Ventral margin with a row of short, curved, spine-like setae, on apex some longer, spine-like setae and a tuft of fine, simple setae. Anterior and posterior surface scattered with stout, lanceolate setae. Patellotibial suture present on basal 1/2 area. Tarsus. Dorsal margin with a row of short, spine-like setae. Ventral margin with a row of curved, spine-like setae. Claw with one row of 10-12 denticles; distally pointed; with 4-5 stripes; subapical setae absent.

Abdomen (Fig. 2c). Surface with dense, irregular rows of U-shaped scale bases. Posterior margin of terga II-IX with triangular spines, approx. as long as wide; spines diminished on middle of posterior margin of tergum IX behind pair of submedian setae. Posterior margin of tergum $\mathrm{X}$ with longer and narrower spines. Posterior margins of sterna VII-IX with shorter triangular spines.

Tergalii (Fig. 2d-f). Present on segments II-VII. Margin with denticles of different sizes, intercalating both medium and long, fine simple setae. Tracheae extending from main trunk to inner and outer margins. Tergalius IV as long as length of segments V and VI combined. Tergalius VII as long as length of segments VIII and 1/2 IX combined.

Paraproct (Fig. 2g). Distally not expanded, with 20-25 stout, marginal spines. Surface scattered with U-shaped scale bases and fine, simple setae. Cercotractor with numerous small, marginal spines.

Subimago (both sexes; Fig. 6d, e). Cuticular coloration. Pronotum brown with lighter areas. Mesonotum brown with medioparapsidal suture contrastingly colorless, other sutures darker brown (Fig. 6d). Thoracic pleura with brown sclerites and colorless membranes (Fig. 6e). Legs mostly colorless; femur colored with brown at base, outer and inner margins, with indistinct brownish spot in distal part; tibia colored with brown at base. Abdominal terga uniformly light brown, sterna lighter. Cerci uniformly colorless.

Hypodermal coloration. As in imago (see below).

Texture. On all legs of both sexes, all tarsomeres covered with blunt microlepides.

Imago, male (Fig. 5a-d, 6c). Head ocher. Turbinate eyes high widened apically, orange. Thorax light brown with ocher areas. Wing membrane colorless, veins ocher; costal brace and adjacent areas with contrasting dark brown stripes. Pterostigma with several oblique veins. Hind wing absent. Legs of all pairs with similar coloration (Fig. 6c): femur light ocher, with inner margin and apex bordered with brown, with reddish macula near apex; tibia with base and apex whitish, other part light brownish on inner side, lighter on 
outer side; tarsus light brownish. Tarsus of middle and hind legs with two apical spines, on segments 1 st +2 nd and 3rd. Abdominal segments I-VI whitish, with peculiar maculae: each tergum II-VII with pair of lateral brown maculae on spiracles; each tergum III-VI with pair of sublateral brown maculae; each tergum III-IV with unpaired reddish stripe on posterior margin and with more or less expressed triangular macula arising from this stripe in anterior direction. Abdominal segments VII-VIII uniformly brown. Abdominal terga IX-X lighter. Each segment of cerci white at base and at apex, contrastingly brown at middle; segments with longer and shorter brown areas irregularly alternating (Fig. 5d).

Male genital structure and development. Imaginal genitals as in Fig. 5e. Sternostyligeral muscle completely absent. Each gonovectis with small hook at apex. Penial bridge without prominent median projection. Unistyligers cylindrical, with straight inner margins. Gonostylus with 1st segment smoothly narrowed toward apex; 2nd segment slightly widened toward apex; 3rd segment short.

Protogonostyli of male larva represent very shallow convexities of posterior margin of abdominal sternum IX. In mature larva ready to molt to subimago, subimaginal gonostyli packed under larval cuticle in «Labiobaetis-pose» (Kluge, 2004: fig. 29I): second segments directed medially and bent (Fig. 5f).

Imago, female (Fig. 6a, b). Head ocher with brownish. Prothorax and anterolateral areas of mesonotum with contrasting light ocher and dark brown markings; other areas of mesonotum and metanotum light brown; ventral side of thorax light uniformly ocher. Wings as in male. Leg coloration as in male. Tarsus of fore leg with two apical spines, on 2nd and 3rd segments (as on middle and hind legs). Each abdominal tergum III and IV with reddish median macula as in male; other markings of abdominal terga and sterna vary individually. Cerci as in male.

Dimension. Body length of larvae $4.2-6.5 \mathrm{~mm}$. Fore wing length (and approximate body length) of subimagos, male and female imagos ca. $5 \mathrm{~mm}$.

Etymology. Refering to the fact that the specimens were collected just behind the campus of Cenderawasih University (UNCEN) in Jayapura.

Distribution. Indonesia: Papua Province (Fig. 7).

Biological aspects. The specimens were collected at an altitude of $160 \mathrm{~m}$ in a medium sized, shallow, moderately flowing river with stones and mud on the bottom, with few vegetation (Fig. 8). The new species was collected together with 12 other mayfly species: four other, yet undescribed species of Labiobaetis Novikova \& Kluge, 1987; 
Mystaxiops venatoris McCafferty \& Sun, 2005; two species of Papuanatula Lugo-Ortiz \& McCafferty, 1999; two species of Centroptella Braasch \& Soldán, 1980; one Cloeon sp.; one Caenis sp. and Nonnullidens reductus Kluge, 2013.

Type-material. Holotype. INDONESIA • larva; Papua Province, Jayapura, Waena, Kamp Wolker; near UNCEN campus; 02³4'07"S, 140³8'51"E; 160 m; 26.v.2019; leg. Surbakti, Kellis \& Sumoked; (PAP080); on slide; GBIFCH00592382; MZB. Paratypes. INDONESIA - 17 larvae; same data as holotype; 3 on slides; GenBank MW041241, MW041242; GBIFCH00673069, GBIFCH00673081, GBIFCH00592381; KSP, MZB; 14 in alcohol; GBIFCH00515503; KSP, MZB • 4 ond 2 imagos with individually associated larval and subimaginal exuviae, $1 q$ subimago with its larval exuviae, 19 imago, $11 \delta$ and 8 larvae ready to molt to subimagoes, 22 larvae; same locality as holotype; 9-13.viii.2012; leg. N. Kluge \& L. Sheyko; Saint-Petersburg State University.

\section{Key to the species of the Labiobaetis claudiae group (larvae)}

1. Paraproct distally expanded (Fig. 4a) L. stagnum

- $\quad$ Paraproct distally not expanded (Figs 2g, 4b) .. 2

2. Labial palp segment II with rather broad protuberance and segment III subquadrangular (Fig. 4f) L. claudiae

- Labial palp segment II with narrow protuberance and segment III slightly pentagonal (Fig. 1h) L. academicus sp. nov.

\section{Genetics}

The genetic distances (K2P) between the species of the $L$. claudiae group are between $20 \%$ and $23 \%$, and therefore much higher than $3.5 \%$, which is generally considered as a likely maximal value for intraspecific divergence (Hebert et al., 2003; Ball et al., 2005; Zhou et al., 2010) (Table 1).

Table 1. Sequenced specimens and genetic distances (COI, Kimura 2-parameter, \%)

\begin{tabular}{llccccc}
\hline & & Specimens catalog \# & GenBank \# & 1 & $\mathbf{2}$ & $\mathbf{3}$ \\
\hline $\mathbf{1}$ & L. academicus sp. nov. & GBIFCH 00673069 & MW041241 & & & \\
$\mathbf{2}$ & L. academicus sp. nov. & GBIFCH 00673081 & MW041242 & 0 & & \\
$\mathbf{3}$ & L. claudiae & GBIFCH 00508144 & MH619479 & 21 & 21 & \\
$\mathbf{4}$ & L. stagnum & GBIFCH 00465168 & MH619491 & 20 & 20 & 23 \\
\hline
\end{tabular}



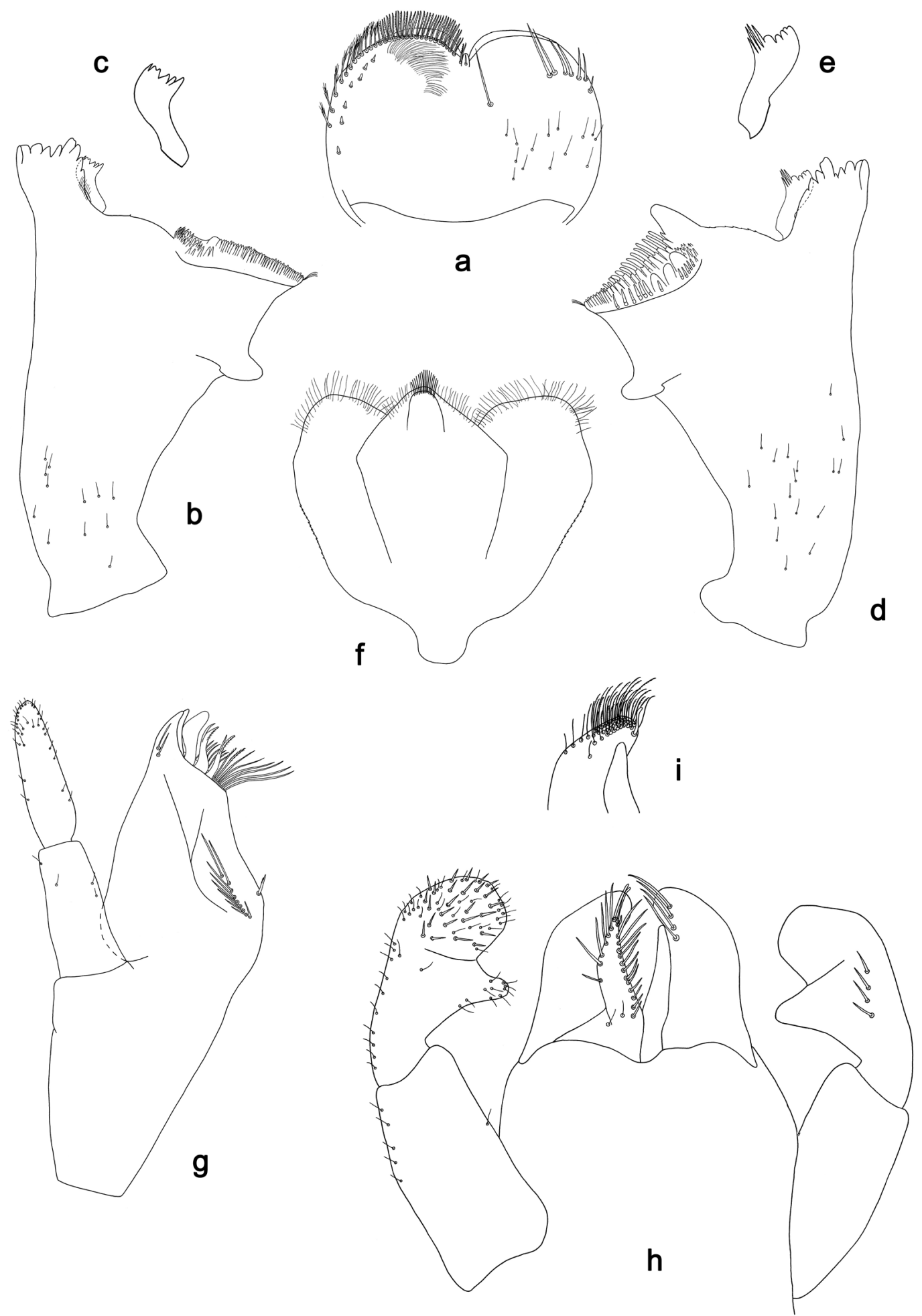

Figure 1. Labiobaetis academicus sp. nov., larva morphology: a Labrum (left, ventral view; right, dorsal view) b Right mandible c Right prostheca d Left mandible e Left prostheca f Hypopharynx and superlinguae g Maxilla h Labium (left, ventral view; right, dorsal view) i Apex of paraglossa. 


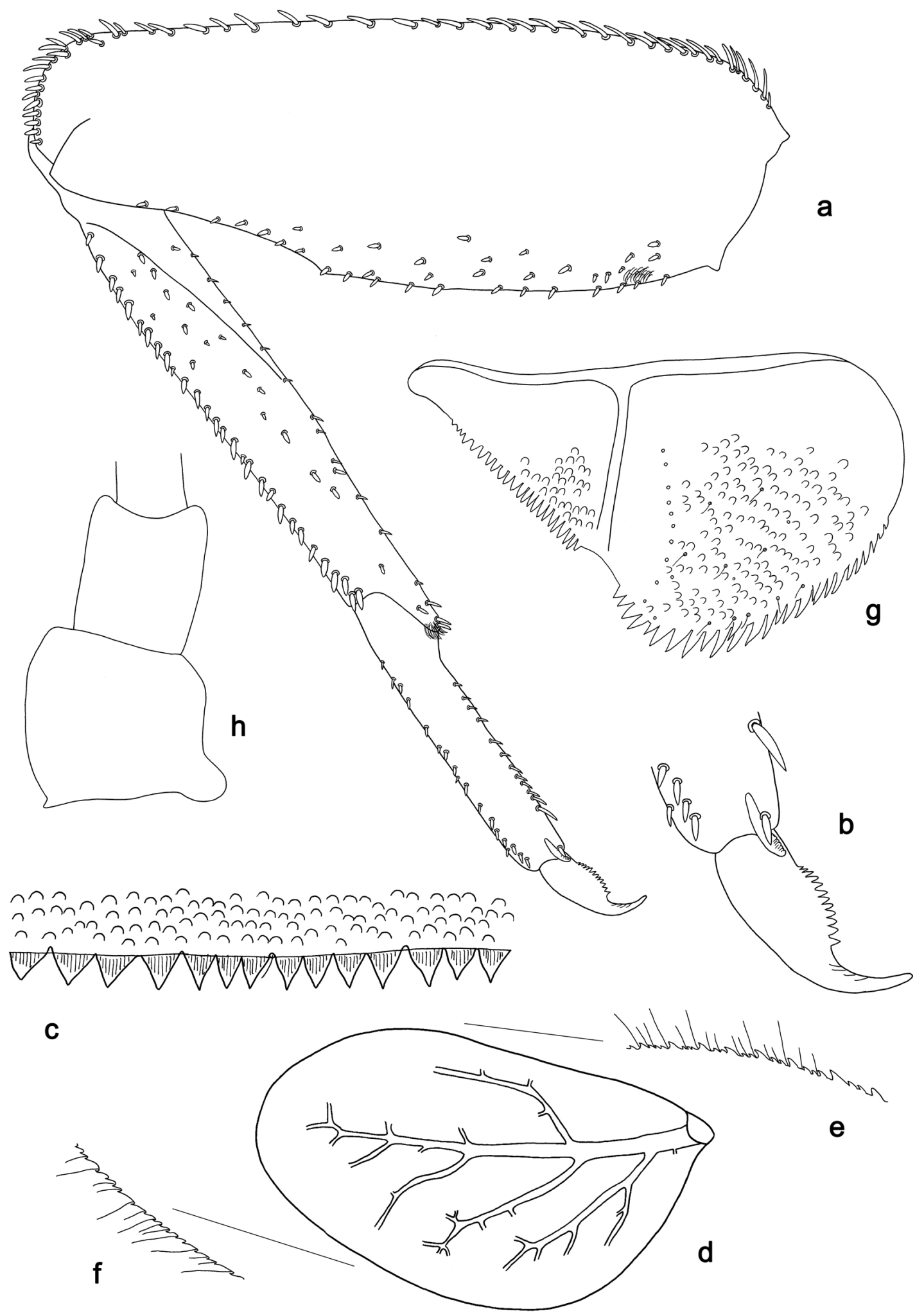

Figure 2. Labiobaetis academicus sp. nov., larva morphology: a Foreleg b Fore claw c Tergum IV $\mathbf{d}$ Tergalius IV e, $\mathbf{f}$ margin of tergalius IV $\mathbf{g}$ Paraproct $\mathbf{h}$ Base of antenna. 
Kaltenbach et al.: Discovery of a new mayfly species ....
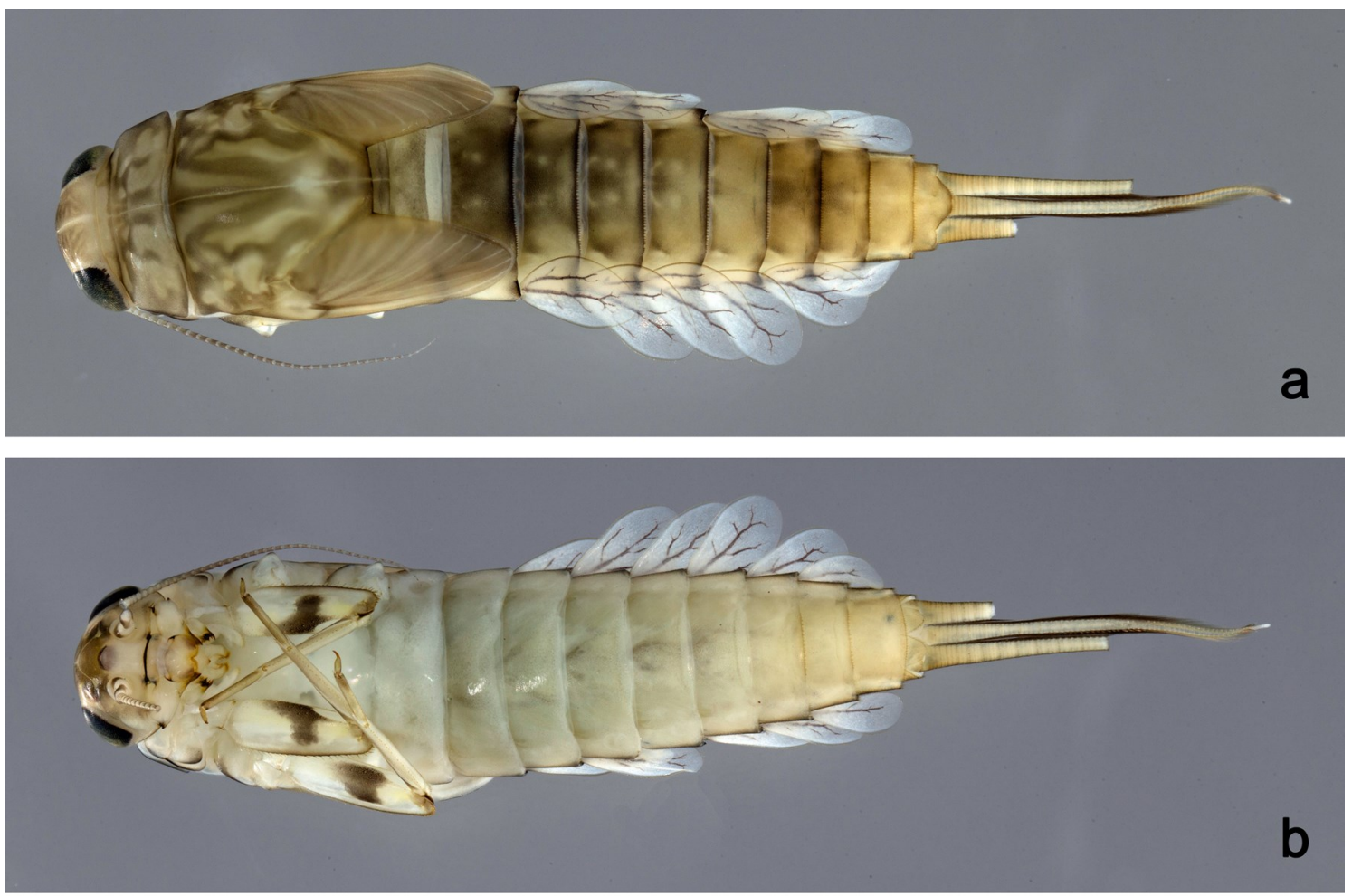

Figure 3. Labiobaetis academicus sp. nov., habitus, larva: a Dorsal view b Ventral view. 

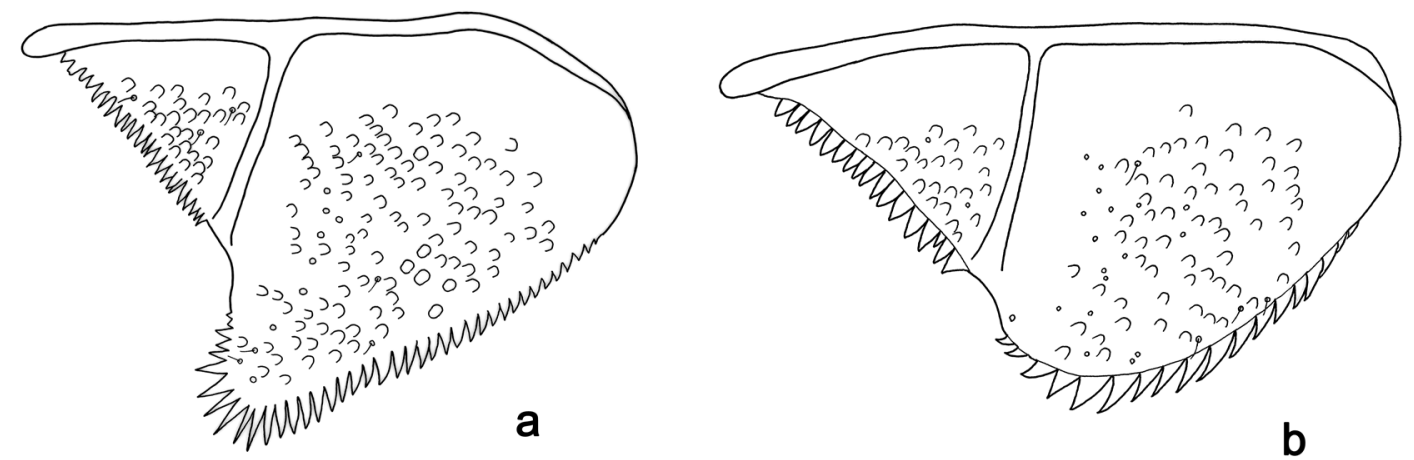

b
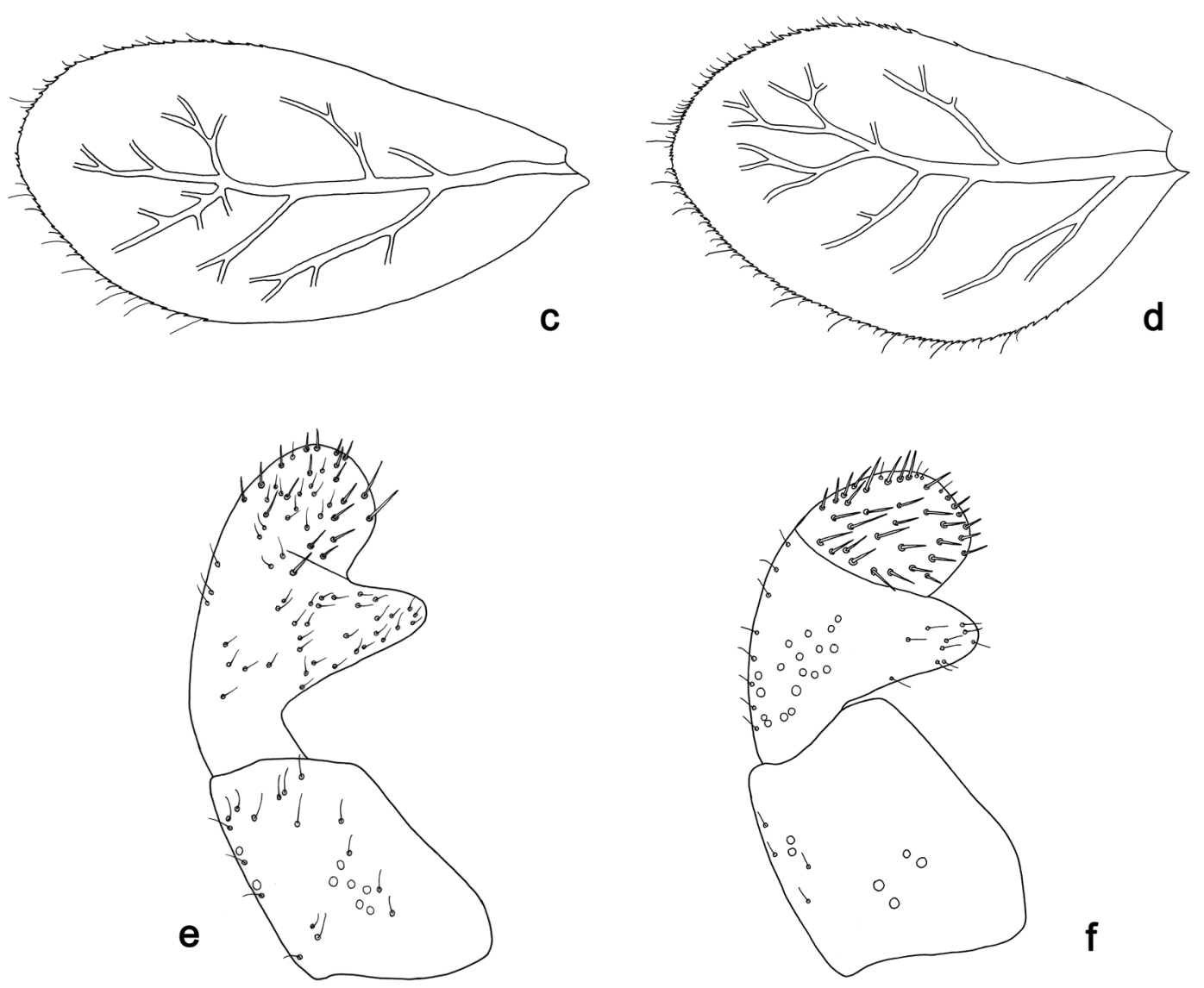

Figure 4. Larvae morphology: a, c, e Labiobaetis stagnum a Paraproct c Tergalius IV e Labial palp; b, $\mathbf{d}, \mathbf{f}$ Labiobaetis claudiae b Paraproct $\mathbf{d}$ Tergalius IV f Labial palp. 

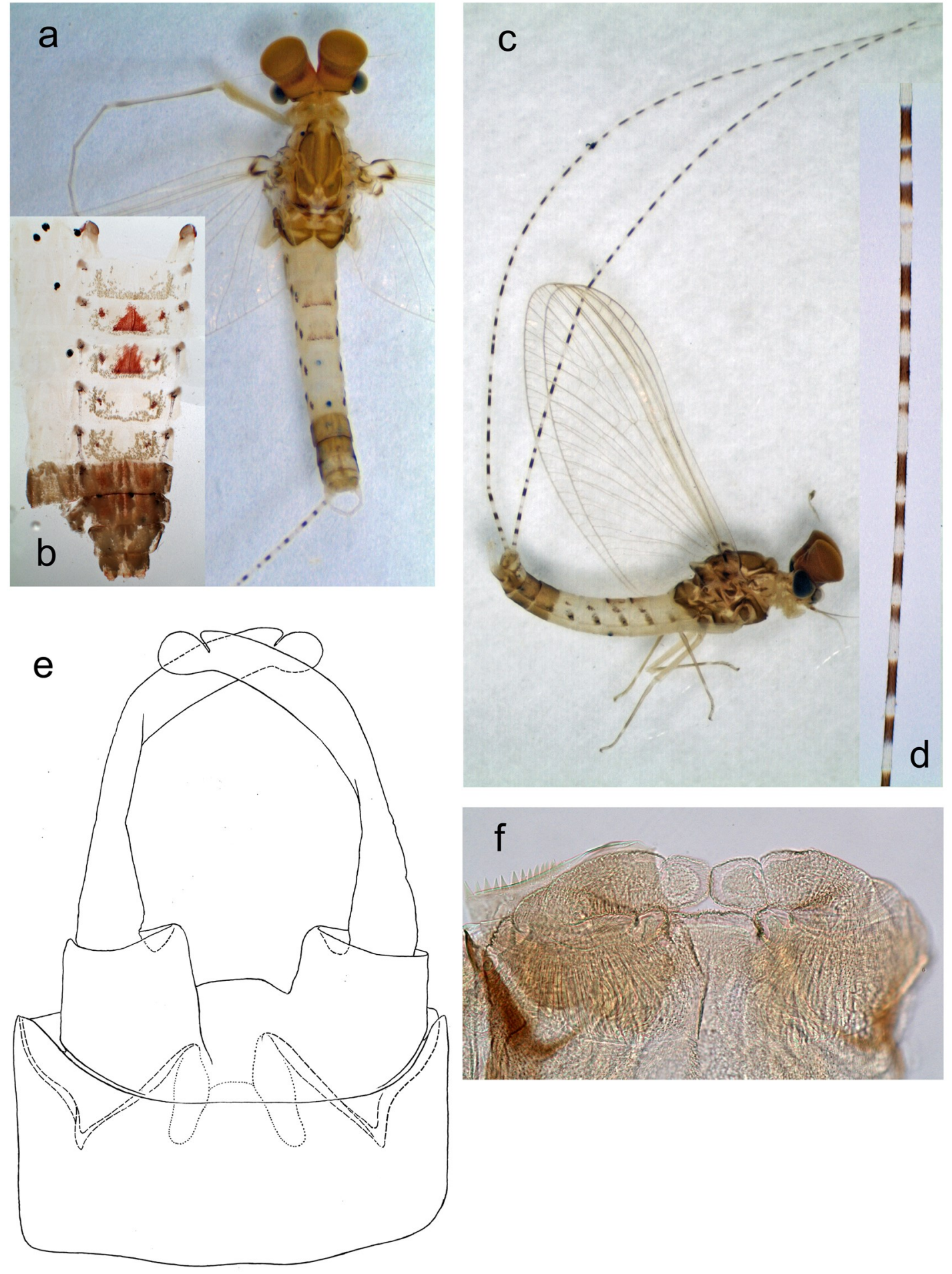

Figure 5. Labiobaetis academicus sp. nov., male imago: a Habitus, dorsal view b Abdomen c Habitus, lateral view d Cercus e Genital structure f Subimaginal gonostyli extracted from mature male larva with fragment of larval protogonostylus. 


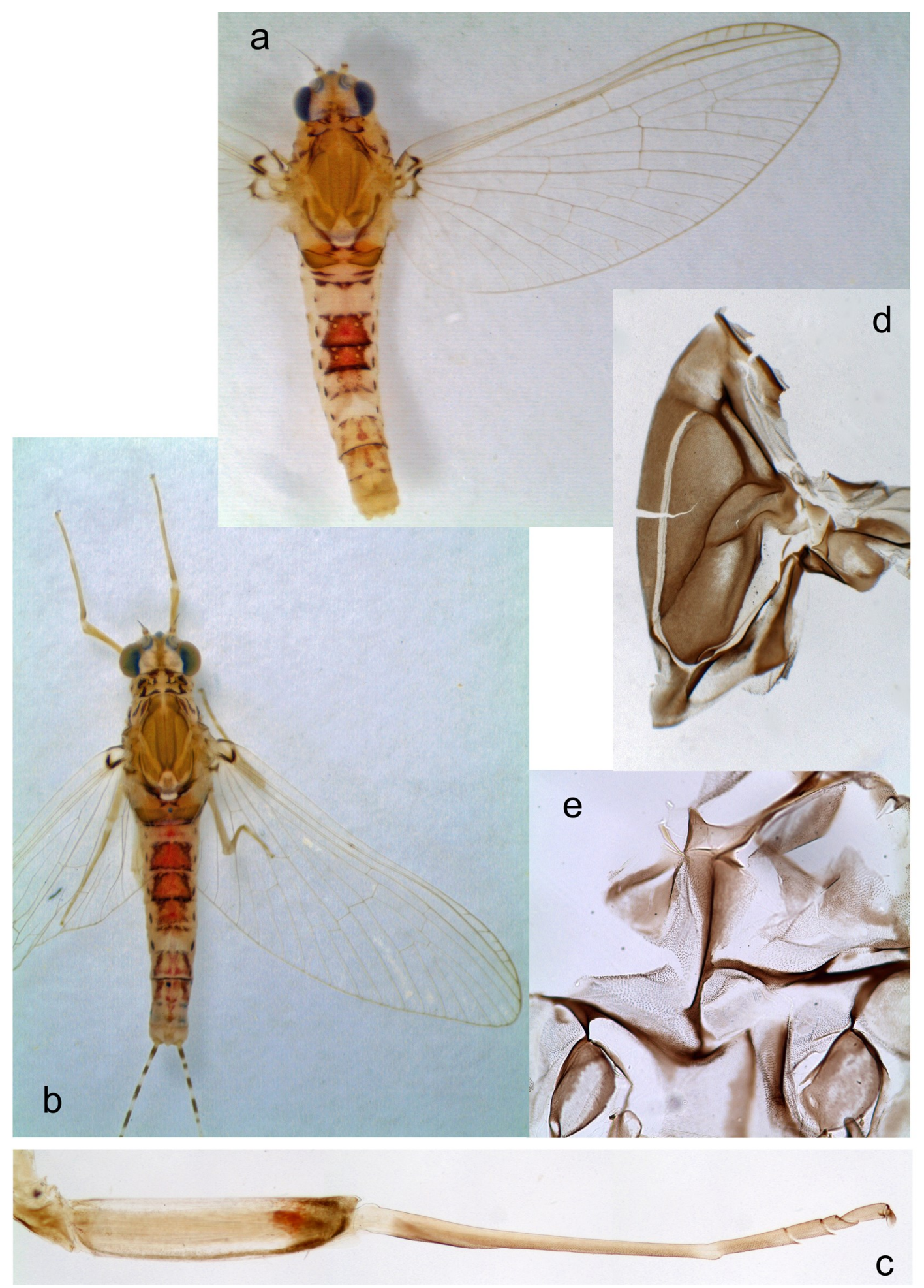

Figure 6. Labiobaetis academicus sp. nov., female imago: a, b Habitus, dorsal view; male imago: c Middle leg; subimago (same in male and female): d Mesonotum e Mesopleuron. 


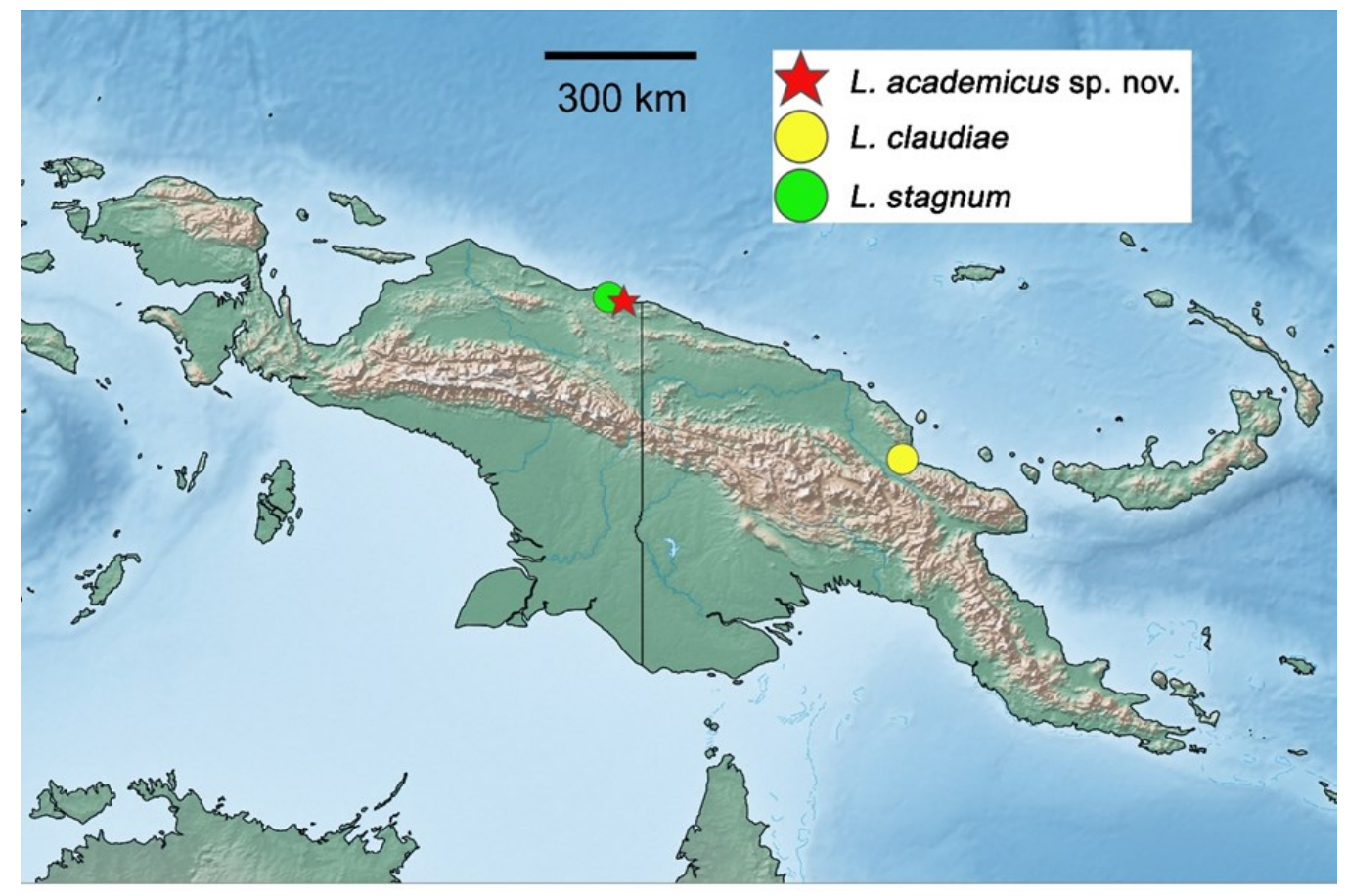

Figure 7. Distribution of Labiobaetis claudiae group in New Guinea.

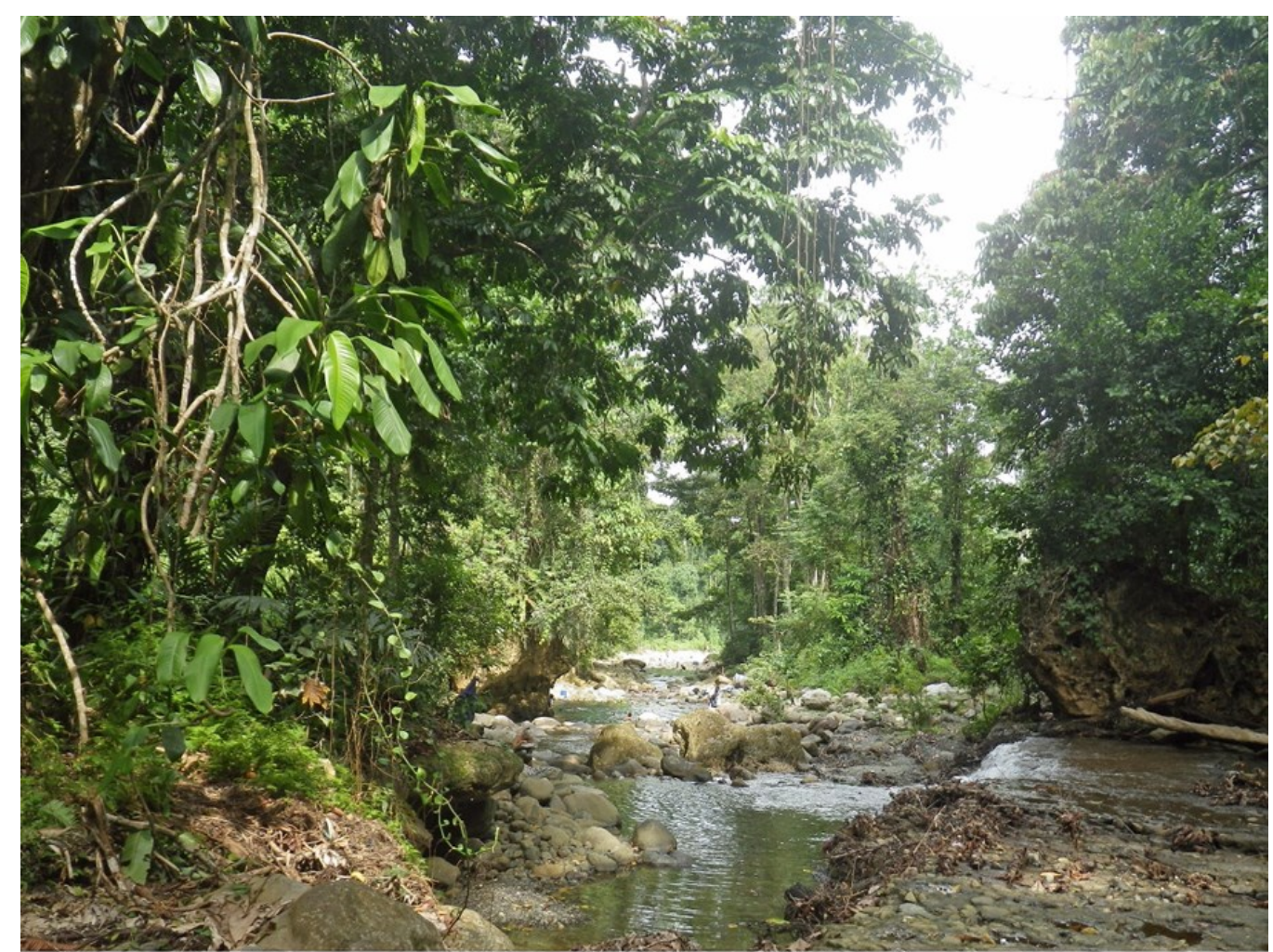

Figure 8. Habitat of L. academicus sp. nov. (photo N.J. Kluge). 


\section{DISCUSSION}

For the assignment of the new species to Labiobaetis we refer to Kluge \& Novikova (2014), and also to Kaltenbach \& Gattolliat (2018; 2019), where the concept of Labiobaetis was summarized and discussed.

Based on the morphological characters as described above, there is no doubt that $L$. academicus sp. nov. belongs to the L. claudiae group and is closely related to L. claudiae and L. stagnum. The morphological groups within Labiobaetis are primarily a working tool but could also serve as a basis for future studies on the generic delimitation and phylogeny of this genus. The inclusion of nuclear gene sequences may prove that some are natural groups.

The genetic distances between the species of the L. claudiae group are rather high, between $20 \%$ and $23 \%$ (K2P, Table 1), which is in line with the genetic distances found between other species in New Guinea (average 22\%; Kaltenbach \& Gattolliat, 2018), Indonesia (11\%-24\%; Kaltenbach \& Gattolliat, 2019) and Borneo (19\%-25\%; Kaltenbach $\&$ Gattolliat 2020). Ball et al. (2005) reported a mean interspecific, congeneric distance of $18 \%$ for mayflies from the United States and Canada.

Astonishingly, the new species was collected in an easily accessible location just behind the Cenderawasih University (UNCEN) campus, together with other mostly undescribed species of mayflies. This underlines that the Indonesian part of the island of New Guinea is still very poorly sampled and that further collections in whatever part of the island will reveal many more unknown species of aquatic insects and Ephemeroptera in particular and especially of Baetidae. Until recently, traditional taxonomic identification posed a serious bottleneck for the sustainable and objective assessment of megadiverse, mostly undescribed faunas. Integrative approaches can help to tackle this issue by flanking taxonomic work with state of the art imaging technology as well as high throughput DNA sequencing. The underlying concepts have been outlined by Balke et al. (2013), with a plea for molecular biodiversity assessment of the Asian freshwater invertebrate fauna. It would be highly justified to develop a focussed national Indonesian collection program for aquatic insects using such approaches in order to obtain a better view on their megadiversity.

\section{ACKNOWLEDGMENTS}

This project is the result of a long-term capacity building program between SNSBZoologische Staatssammlung and Cenderawasih University (UNCEN) (Research Permit Nr. 345/E5/E5.4/SIP/2019). We very much acknowledge support from the German Research 
Foundation DFG which provided the opportunity for training of Dr. Surbakti in Munich. This project was also supported by SNSB Innovativ scheme funded by the Bayerisches Staatsministerium für Wissenschaft und Kunst.

We sincerely thank the management of Cenderawasih University, who made our study possible and the collection team for their successful effort. We are also grateful to Marion Podolak (MZL) and Maud Liégeois (University of Lausanne, UNIL) for their support with lab work and preparation of the COI barcodes. Lastly, the authors are thankful to the reviewers for the recommendations and comments on the manuscript.

\section{REFERENCES}

Ball, S.L., Hebert, P.D.N., Burian, S.K. \& Webb, J.M. 2005. Biological identifications of mayflies (Ephemeroptera) using DNA barcodes. Journal of the North American Benthological Society, 24: 508-524. https://doi.org/10.1899/04-142.1

Balke, M., Hendrich, L., Toussaint, E.F., Zhou, X., von Rintelen, T. \& De Bruyn, M. 2013. Suggestions for a molecular biodiversity assessment of South East Asian freshwater invertebrates. Lessons from the megadiverse beetles (Coleoptera). Journal of Limnology, 72: 61-68. https:// doi.org/10.4081/jlimnol.2013.s2.e4

Cruz, P.V., Nieto, C., Gattolliat. J-L., Salles, F.F. \& Hamada, N. 2020. A cladistic insight into higher level classification of Baetidae (Insecta: Ephemeroptera). Systematic Entomology, 2020: 1-12. https://doi.org/10.1111/syen.12446

Folmer, O., Black, M., Hoeh, W., Lutz, R. \& Vrijenhoek, R. 1994. DNA primers for amplification of mitochondrial cytochrome $\mathrm{c}$ oxidase subunit I from divers metazoan invertebrates. Molecular Marine Biology and Biotechnology, 3: 294-299. http://www.mbari.org/staff/vrijen/PDFS/ Folmer_94MMBB.pdf

Gattolliat, J-L. \& Nieto, C. 2009. The family Baetidae (Insecta: Ephemeroptera): synthesis and future challenges. A quatic Insects, 31: 41-62. https://doi.org/10.1080/01650420902812214

Hebert, P.D.N., Cywinska, A., Ball, S.L. \& DeWaard, J.R. 2003. Biological identifications through DNA barcodes. Proceedings of The Royal Society B-Biological Sciences, 270: 313-321. https:// doi.org/10.1098/rspb.2002.2218

Hubbard, M.D. 1995. Towards a standard methodology for the description of mayflies (Ephemeroptera). In: Corkum, L.D. \& Ciborowski, J.J.H. (Eds). Current directions in research on Ephemeroptera. Canadian Scholar's Press, Toronto, 361-369.

Jacobus, L.M., Macadam, C.R. \& Sartori, M. 2019. Mayflies (Ephemeroptera) and their contributions to ecosystem services. Insects, 10: 1-26. https://doi.org/10.3390/insects 10060170

Kaltenbach, T. \& Gattolliat, J-L. 2018. The incredible diversity of Labiobaetis Novikova \& Kluge in New Guinea revealed by integrative taxonomy (Ephemeroptera, Baetidae). ZooKeys, 804: 1-136. https://doi.org/10.3897/zookeys.804.28988

Kaltenbach, T. \& Gattolliat, J-L. 2019. The tremendous diversity of Labiobaetis Novikova \& Kluge in Indonesia (Ephemeroptera, Baetidae). ZooKeys, 895: 1-117. https://doi.org/10.3897/ zookeys.895.38576

Kaltenbach, T. \& Gattolliat, J-L. 2020. Labiobaetis Novikova \& Kluge in Borneo (Ephemeroptera, Baetidae). ZooKeys, 914: 43-79. https://doi.org/10.3897/zookeys.914.47067

Kaltenbach, T. \& Gattolliat, J-L. 2021a. Labiobaetis Novikova \& Kluge in Ethiopia (Ephemeroptera, Baetidae), with description of a new species. African Invertebrates, 62: 231-255. https:// doi.org/10.3897/AfrInvertebr.62.62029 
Kaltenbach, T. \& Gattolliat, J-L. 2021b. Labiobaetis Novikova \& Kluge in West Africa (Ephemeroptera, Baetidae), with description of a new species. African Invertebrates (in press).

Kaltenbach, T., Garces, J.M. \& Gattolliat, J-L. 2020. The success story of Labiobaetis Novikova \& Kluge in the Philippines (Ephemeroptera, Baetidae), with description of 18 new species. ZooKeys (in press).

Kimura, M. 1980. A simple method for estimating evolutionary rates of base substitutions through comparative studies of nucleotide sequences. Journal of Molecular Evolution, 16: 111-120. https://doi.org/10.1007/BF01731581

Kluge, N.J. 2004. The phylogenetic system of Ephemeroptera. Academic Publishers, Dordrecht, 1442.

Kluge, N.J. \& Novikova, E.A. 2014. Systematics of Indobaetis Müller-Liebenau \& Morihara 1982, and related implications for some other Baetidae genera (Ephemeroptera). Zootaxa, 3835: 209236. http://dx.doi.org/10.11646/zootaxa.3835.2.3

Kumar, S., Stecher, G. \& Tamura, K. 2016. MEGA 7: molecular evolutionary genetics analysis version 7.0 for bigger data sets. Molecular Biology and Evolution, 33: 1870-1874. https:// doi.org/10.1093/molbev/msw054

Lugo-Ortiz, C.R., McCafferty, W.P. \& Waltz, R.D. 1999. Definition and reorganization of the genus Pseudocloeon (Ephemeroptera: Baetidae) with new species descriptions and combinations. Transactions of the American Entomological Society, 125: 1-37.

Novikova, E.A. \& Kluge, N.J. 1987. Systematics of the genus Baetis (Ephemeroptera, Baetidae), with descriptions of new species from Middle Asia. Vestnik Zoologii, 1987(4): 8-19 (in Russian).

Sanger, F., Nicklen, S. \& Coulson, A.R. 1977. DNA sequencing with chain-terminating inhibitors. Proceedings of the National Academy of Sciences U.S.A., 74: 5463-5467. https://doi.org/10.1073/ pnas. 74.12 .5463

Sartori, M. \& Brittain, J.E. 2015. Order Ephemeroptera. In: Thorp, J. \& Rogers, D.C. (Eds) Ecology and general biology: Thorp and Corvich's Freshwater Invertebrates. Academic Press, 873-891. https://doi.org/10.1016/B978-0-12-385026-3.00034-6

Shi, W. \& Tong, X. 2014. The genus Labiobaetis (Ephemeroptera: Baetidae) in China, with description of a new species. Zootaxa, 3815: 397-408. http://dx.doi.org/10.11646/ zootaxa.3815.3.5

Shorthouse, D.P. 2010. SimpleMappr, an online tool to produce publication-quality point maps. [Retrieved from https://www.simplemappr.net. Accessed July 03, 2020].

Tofilski, A. 2018. DKey software for editing and browsing dichotomous keys. ZooKeys, 735: 131140. https://doi.org/10.3897/zookeys.735.21412

Vuataz, L., Sartori, M., Wagner, A. \& Monaghan, M.T. 2011. Toward a DNA taxonomy of Alpine Rhithrogena (Ephemeroptera: Heptagenidae) using a mixed Yule-Coalescent Analysis of mitochondrial and nuclear DNA. PLOS ONE, 6: 1-11. https://doi.org/10.1371/ journal.pone. 0019728

Zhou, X., Jacobus, L.M., DeWalt, R.E., Adamowicz, S.J. \& Hebert, P.D.N. 2010. Ephemeroptera, Plecoptera, and Trichoptera fauna of Churchill (Manitoba, Canada): insights into biodiversity patterns from DNA barcoding. Journal of the North American Benthological Society, 29: 814837. https://doi.org/10.1899/09-121.1 